[Feb., 1903.] THE BOUNDARY IN CONFORMAL MAPPING. 233

\title{
ON THE TRANSFORMATION OF THE BOUNDARY IN THE CASE OF CONFORMAL MAPPING.
}

\author{
BY PROFESSOR W. F. OSGOOD.
}

(Read before the American Mathematical Society, December 30, 1902.)

THE theorem that the interior of any simply connected region may be mapped in a one-to-one manner and conformally on the interior of a circle was stated by Riemann in his doctor's dissertation and was proved by Schwarz and Neumann for the case that the boundary consists of a curve subject to certain restrictions. In the first volume of the Transactions * I extended this theorem to the most general simply connected plane region. The generalization is remarkable in that the boundary of such a region need not be a curve, but may be a set of points some of which can not be approached along any curve lying wholly in the region. An extension of the definition of a simply connected region is here obviously necessary. Such a definition may be laid down as follows: By a simply connected region of a plane is meant a continuum such that the whole interior of any closed Jordan curve drawn in the region lies in the region. By a "Jordan curve" is meant a continuous curve without multiple points.

The further question presents itself as to whether boundary points of the region are carried over continuously into points of the circumference of the circle. This question I am able to answer by means of the following theorems. The given simply connected region is denoted by $S$ and it is mapped conformally on the interior of the circle $\widetilde{S}$.

Theorem I. Let $A$ be a point of the boundary of $S$ which can be approached along a curve $C$ lying wholly in $\$$. Then the image of $C$ in $\mathbb{S}$ is a curve $\mathbb{S}$ abutting on a single point $\mathfrak{A}$ of the circumference of $\mathfrak{S}$; so that, if the point $P$ approaches $A$ along $C$, the image $\mathfrak{P}$ of $P$ in $\mathfrak{S}$ will approach $\mathfrak{A}$ along $\mathfrak{E}$.

Theorem II. Let $C^{\prime}$ be a second curve of $S$ also leading to $A$, but meeting $C$ only in the initial point of $C$ and in the point

* "On the existence of the Green's function for the most general simply connected plane region," Transactions Amer. Math. Soc., vol. 1 (1900), p. 310. 
A. Then the image of $C^{\prime}$ in $\mathbb{S}$ will be a curve $\mathbb{C}^{\prime}$ abutting on a point $\mathfrak{A}$ ' of the circumference of $\mathfrak{S}$. The necessary and sufficient condition that $\mathfrak{U}^{\prime}$ coincide with $\mathfrak{A}$ is that the closed curve consisting of the two arcs $C, C^{\prime}$ may be drawn together continuously to the point $A$ without passing out of the region $S$; in other words, that this closed curve shall contain in its interior only points of $S$.

Note that $S$ consists exclusively of interior points, the boundary not being counted as belonging to the region.

Theorem III. If $A, A^{\prime}$ are two distinct points of the boundary of $S$ which can be approached along a curve $C$ or $C^{\prime}$ respectively, then the corresponding points $\mathfrak{A}, \mathfrak{A}^{\prime}$ on the circumference of $\subseteq$ will be distinct.

Theorem IV. Let $B$ be a point of the boundary of $S$, such that a set of points $Q_{1}, Q_{2}, \ldots$ can be chosen in $S$ which have $B$ as their sole limiting point:

$$
\lim _{n=\infty} Q_{n}=B ;
$$

but through which no curve can be passed, lying wholly in $S$ and such that a point $P$ describing this curve will approach $B$ as its limit. Suppose furthermore that a curve $D$ can be drawn in $S$ passing through the points $Q_{n}$ and having but one limiting point $A$ on the boundary of $S$ which can be approached along a curve $C$, cutting $D$ in every neighborhood of $A$. Then if the point $P$ describe D, the image $\mathfrak{P}$ of $P$ in $\subseteq$ will approach the point $\mathfrak{A}$ of the boundary of $\subseteq$ which corresponds to $A$.

Theorem $\mathrm{V}$. In particular, let $S$ be a region having no boundary points of the kind B considered in Theorem IV. Then to each point of the circumference of $\mathbb{\subseteq}$ will correspond a single point of the boundary of $S$ in this sense that if a point $\mathfrak{B}$ approach a point $\mathfrak{A}$ of this circumference along a curve of $\mathbb{S}$, the image $P$ of $\mathfrak{P}$ in $S$ will approach a point $A$ of the boundary of $S$, and $A$ will be the same point for all curves of $\subseteq$ leading to $\mathfrak{A}$. But to distinct points $\mathfrak{A}, \mathfrak{A}^{\prime}$ may correspond the same point $A$.

The boundary of $S$ in this case consists of a curve whose coördinates may be represented as single valued continuous functions of a parameter $t$

$$
x=f(t), \quad y=\phi(t),
$$

where $t$ may be chosen as the arc of the circumference of $\mathbb{S}$, and $f(t), \phi(t)$ are periodic with the length of the circumference of $\mathfrak{S}$ as period. 
If furthermore it is impossible to draw a Jordan curve all of whose points but one lie in $S$ and which contains in its interior points of the boundary of $S$, then the boundary points of $S$ will form a Jordan curve.

The result stated in the latter part of this theorem was recently published by Schoenflies.* In the general case the boundary may be considered as forming a generalization of the Jordan curve; for, although the boundary may pass through a given point more than once, it does not cut itself, and it forms the boundary of a simply connected region.

The method on which the proof of these theorems rests was suggested by physical evidence. The conformal map of $S$ on $\mathbb{S}$ is effected by the Green's function $g$ of the region $S$, with pole at $O$, the existence of $g$ having been established in my former paper $(l . c$.$) . This function may be interpreted in the well-$ known manner as the temperature in a certain flow of heat or as the potential in the analogous flow of electricity, the source being at $O$. The function $h$ conjugate to $g$ can be employed to measure the flux across a given curve; in fact, if $c$ denote an arc drawn in $S$, the flux across $c$ is proportional to the difference in the values of $h$ at the extremities of $c$. I was led to the theorems stated above through the following

Physical Law. - Let a fixed circle be drawn in $S$ with $O$ as centre. Let c be an open curve of length $l$ drawn in $S$, but lying outside the fixed circle. Let the maximum numerical value of the flux across c (or the upper limit, in case no maximum exists), when $c$ assumes all possible forms and positions, be denoted by $f(l)$. Then

$$
\lim _{l=0} f(l)=0 .
$$

A rigorous proof of this law may be given by means of the theory of the logarithmic potential function, and proofs of all the theorems here stated will appear later.

Harvard UnIVERSity, Cambridae,

17 December, 1902.

* "Ueber einen grundlegenden Satz der Analysis situs," Göttinger Nachrichten, 1902, Heft 2. 